\title{
Damage to rigid-particle-filled polymer under cyclic tension
}

\author{
Shu-Lin Bai, ${ }^{1,2 *}$ Zong-De Liü ${ }^{3}$ and Yang Ju${ }^{4}$ \\ ${ }^{1}$ State Key Laboratory of Non-linear Mechanics (LNM), Institute of Mechanics, Chinese Academy of Sciences, Beijing, 100080, People's \\ Republic of China \\ ${ }^{2}$ Key Laboratory of Polymeric Composite and Functional Materials, Ministry of Education of China, Guangzhou 510275, People's Republic \\ of China \\ ${ }^{3}$ Department of Power Engineering North China Electric Power University, Beijing 102206, People's Republic of China \\ ${ }^{4}$ Division of Mechanics and Structural Engineering, Testing Centre for Materials and Mechanics, CUMTB-Shimadzu, China University of \\ Mining and Technology, D11, Xueyuan Road, Beijing 100083, People's Republic of China
}

\begin{abstract}
The effect of particle volume fraction, strain rate and interfacial bonding strength on the damage to a glass bead-filled high density polyethylene composite was studied experimentally by means of cyclic tension tests. Although the volume fraction of the glass beads varies from $5 \%$ to $25 \%$, the materials studied are always sensitive to the strain rate, reflected by an increase of the modulus with strain rates changing from $10^{-3} \mathrm{~s}^{-1}$ to $10^{-5} \mathrm{~s}^{-1}$. The damage evolution is a function of the applied far-field strain, but is also strongly influenced by the particle volume fraction, strain rate and interfacial bonding strength. Strong interfacial adhesion can postpone the initiation of damage and cause a lower level of damage than that occuring with weak adhesion under a given strain. For the studied materials with interfacial debonding taking place, the higher the volume fraction of the glass beads, the greater the number of microcracks formed at the interface. The effect of strain rate on the damage may be related to the effect of loading time, ie low strain rate favours damage development. The residual strain is a function of far-field strain and also depends on the strain rate and residual deformation of microvoids formed at two poles of the particle.

(C) 2001 Society of Chemical Industry
\end{abstract}

Keywords: rigid particle filled polymer; cyclic tension; damage; affecting factors

\section{INTRODUCTION}

The concept of adding rigid particles into a tough polymer was first raised in $1984^{1}$ in the hope of increasing both its strength and toughness. In general, the strengthening effect can be relatively easily obtained with the above material systems. The toughening effect depends quite strongly on factors such as the particle size and size distribution, volume fraction and distribution of the particles, and strength of the interfacial adhesive bond. Both strength and ductility are related to the mechanisms causing damage. Therefore, the development of damage in rigidparticle-filled polymers is also controlled by the factors cited above.

Adhesion at the particle-polymer interface has been shown to exert a considerable influence on the mechanical responses, and a correlation between the characteristics of the particles and the polymer properties has been established. ${ }^{2}$ If the interfacial adhesion is strong, interfacial debonding will be initiated at a relatively high stress level. Otherwise, it will take place at low stress and so the damage will occur too early. This was proved in our previous work ${ }^{3,4}$ with a glass- bead-filled high density polyethylene. The polymer and rigid-particle-filled polymer are generally viscoelastic or viscoplastic materials, and their mechanical properties are sensitive to strain rates. This can be seen from the dependence of the Young modulus and maximum tensile stress on the strain rates. ${ }^{5}$ While the damage rate for ductile materials is proportional to the cumulative strain rate and the released strain energy density ${ }^{6}$ in the case of strong adhesion at the interface, both tensile strength and elastic modulus increase with the particle volume fraction, and the modulus is also affected by the particle size. ${ }^{7}$ Therefore, the damage to a rigid-particle-filled polymer depends on many factors including the microstructure and properties of the particle and matrix.

In this paper, three factors are studied experimentally: interfacial adhesive strength, strain rate, and volume fraction of the particles. The material system used is glass bead (GB)-filled high density polyethylene (HDPE). The results obtained certainly help us to understand the damage mechanisms of a rigidparticle-filled polymer. It should be mentioned that a similar study concerning the pristine HDPE used in

* Correspondence to: Shu-Lin Bai, Department of Mechanics and Engineering Science, Peking University, Beijing 100871, People's Republic of China

E-mail: bai@mech.pku.edu.cn

Contract/grant sponsor: National Natural Science Foundation of China; contract/grant number: 19872007; contract/grant number: 10032010

(Received 24 October 2000; revised version received 28 February 2001; accepted 12 April 2001) 
this study is reported in refs 4 and 5. A comparison of mechanical properties between pure HDPE and glassbead-filled HDPE is also presented in the same references. Therefore, no results on pure HDPE have been introduced in this work.

\section{MATERIALS AND TEST METHODS}

The HDPE used was supplied by the Da-Qing Oil Plant of China and the glass beads by the Glass Company of Beijing, China. HDPE is a semicrystalline thermoplastic material. Its crystallinity may be as high as $72.1 \%{ }^{8}$ Before blending with HDPE, the GB were dried in an oven at $110^{\circ} \mathrm{C}$ for $12 \mathrm{~h}$. The GBs were firstly precoated separately with two coupling agents: silane (A-1100, $\mathrm{H}_{2} \mathrm{~N}\left(\mathrm{CH}_{2}\right)_{3} \mathrm{Si}\left(\mathrm{OC}_{2} \mathrm{H}_{5}\right)_{3}$ and titanate $\left(\mathrm{NDZ}-101,\left(\mathrm{CH}_{3}\right)_{2} \mathrm{CHOTi}\left[\mathrm{OPO}\left(\mathrm{OC}_{8} \mathrm{H}_{17}\right)_{2}\right]_{3}\right)$ with surfactant content of $1 \mathrm{wt} \%$. The treated GBs were blended with HDPE in a twin-screw extruder at 200$220^{\circ} \mathrm{C}$, the feeder and screw speeds are 30 Watt and 180 Watt respectively. For A-1100 treated GB/HDPE composites, to increase interfacial adhesion, $10 \mathrm{wt} \%$ of HDPE- $g$-maleic acid was added. The pelleted extrudate was injection moulded into tensile samples. Before mixing, the exact weight ratios between HDPE and GB coupled with coupling agent were chosen, so that the weight fractions of GB were known; the GB volume fractions were calculated using the relationship between the weight and volume, the density of all materials being known. Three weight ratios were chosen to give three GB volume fractions of $5 \%$, $15 \%$ and $25 \%$, respectively. The diameter of $500 \mathrm{~GB}$ beads was measured; they varied from about 15 to $45 \mu \mathrm{m}$, with the maximum percentage at about $30 \mu \mathrm{m}$, as shown in Fig 1.

Table 1 gives the coupling agents used and the interfacial bonding states of the materials studied. Chemical bonding was assumed to have greater bonding strength than physical entanglement. In fact, a study on the same material systems shows that the interfacial tensile strength of HDPE/GB coated with silane was about $17.2 \mathrm{MPa}$, while that treated with titanate was about $8.6 \mathrm{MPa} .{ }^{9}$ The modification of

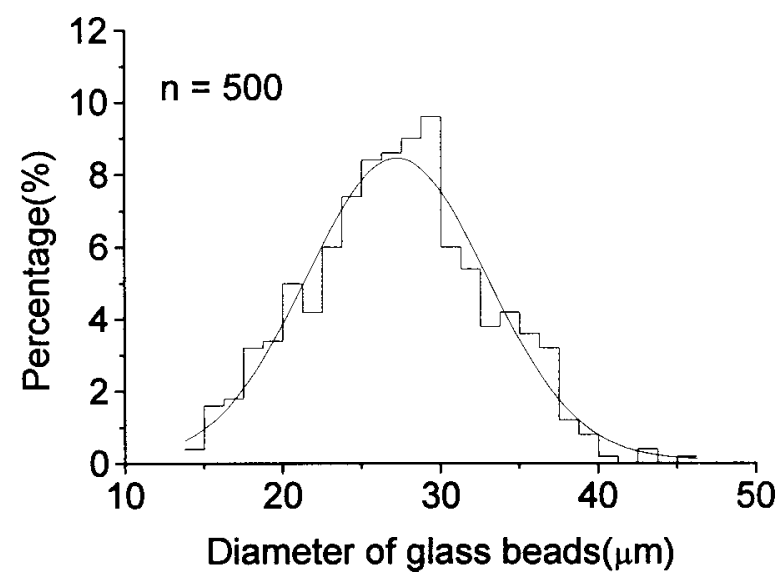

Figure 1. Size distribution of glass beads.
Table 1. Glass bead/HDPE systems used in the study

\begin{tabular}{lccc}
\hline Materials & $\begin{array}{c}\text { Volume } \\
\text { fraction (\%) }\end{array}$ & $\begin{array}{c}\text { Coupling } \\
\text { agents }\end{array}$ & $\begin{array}{c}\text { Interfacial } \\
\text { state }\end{array}$ \\
\hline GH4 & 5 & 1wt\% Titanate & Physical \\
GH5 & 15 & & entanglement \\
GH6 & 25 & & \\
GH7 & 5 & 1wt\% Silane & Chemical \\
GH8 & 15 & $+10 w t \%$ HDPE-g-MA & bonding \\
GH9 & 25 & & \\
\hline
\end{tabular}

particle surfaces may be brought about in a variety of ways. ${ }^{10}$ One can obtain different structures for surface monolayers on particle surfaces depending on bulk and functionality of the absorbing molecule and the

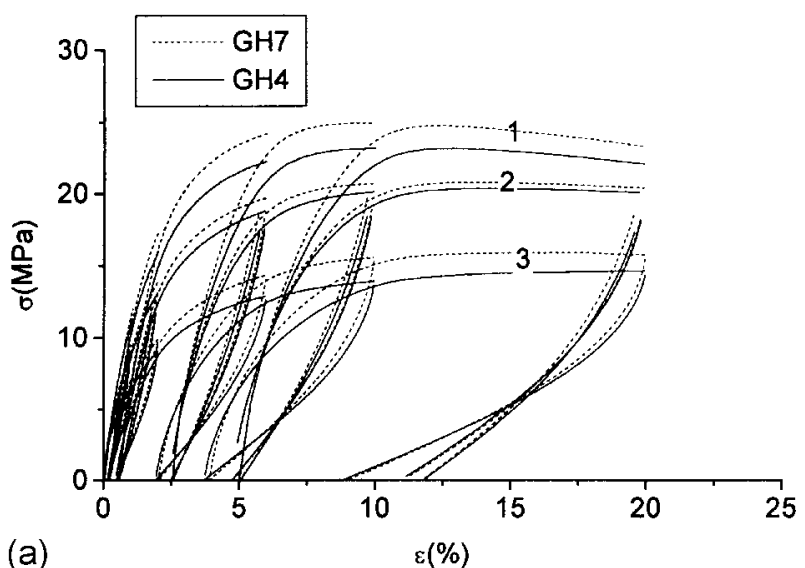

(a)

$\varepsilon(\%)$
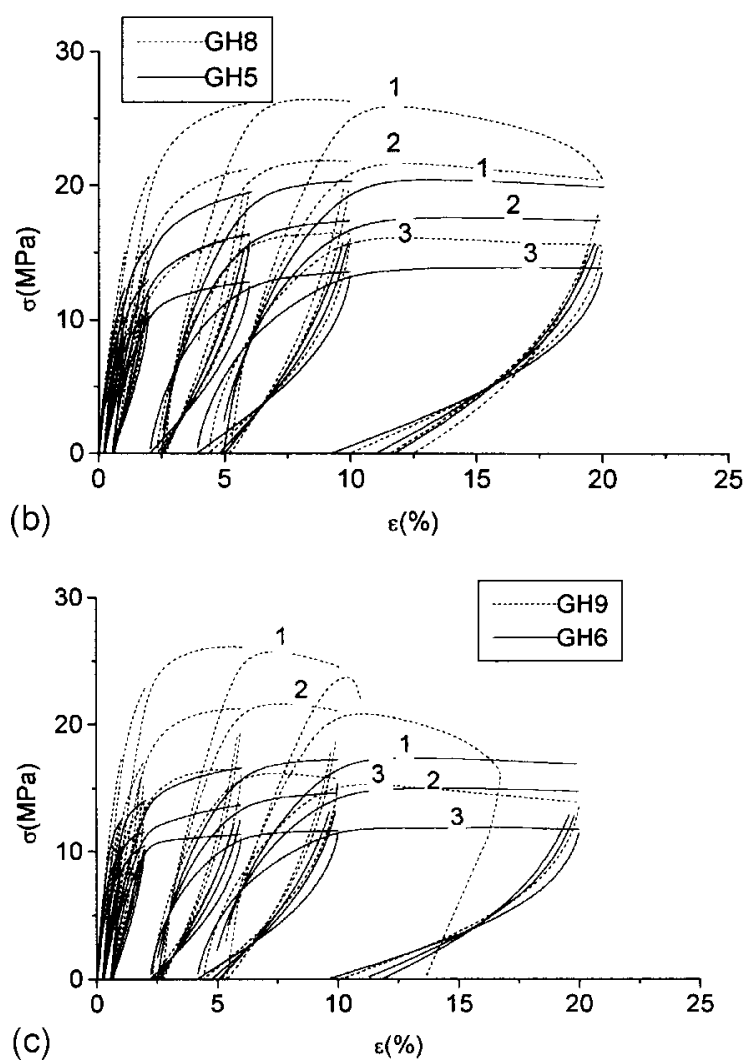

Figure 2. Cyclic tensile $\sigma-\varepsilon$ curves: a, $8 \times 10^{-3} \mathrm{~s}^{-1}$; b, $8 \times 10^{-4} \mathrm{~s}^{-1}$; c, $3 \times 10^{-5} \mathrm{~s}^{-1}$. 
density of surface reactive sites. ${ }^{9}$ The interfacial connection between coated GB and HDPE was illustrated by Bai et $a l^{4}$ for the materials studied in this work.

The cyclic tensile tests were carried out on an MTS810 instrument under strain rates of $8 \times 10^{-3} \mathrm{~s}^{-1}$, $8 \times 10^{-4} \mathrm{~s}^{-1}$ and $3 \times 10^{-5} \mathrm{~s}^{-1}$, respectively. The geometry of the tensile samples was determined by ASTM D638M. From the cyclic $\sigma-\varepsilon$ curves, the Young moduli were measured and the damage evolution evaluated.

\section{RESULTS AND DISCUSSION}

3.1 Stress-strain relationship under cyclic tension The cyclic $\sigma-\varepsilon$ curves obtained are shown in Fig 2. The unloading process continues until zero load for each cycle, and then reloading follows. In general, polymer materials such as HDPE show viscoelastic behaviour, ie the stress depends on both strain and strain rates. The viscoelastic properties of the materials studied are demonstrated by the fact that $\sigma-\varepsilon$ curves move up with increasing strain rates. Strain softening happens at a strain magnitude of about $10 \%$ under a strain rate of $8 \times 10^{-3} \mathrm{~s}^{-1}$, marked by stress decreasing when the strain exceeds $10 \%$, while under lower strain rates, the maximum tensile stress remains almost constant, meaning that no strain softening phenomenon happens. For the composite with $25 \%$ of GB by volume, some samples were ruptured before the farfield strain reached $20 \%$. It seems from this phenomenon that the fracture strain decreases with increasing GB volume fraction. This result means that toughness decreases with increasing GB content. In fact, the toughness depends on many factors, such as particle size, particle content and interfacial adhesion strength. One important parameter is the thickness of the ligament between two particles; under a critical ligament thickness, the toughening effect can be greatly enhanced. Many studies have considered this problem. ${ }^{11,12}$

The role of the interface on the strengthening effect can also be seen from the $\sigma-\varepsilon$ curves in Fig 2. The tensile $\sigma-\varepsilon$ curves for $\mathrm{GH} 7-9$ with strong interfacial

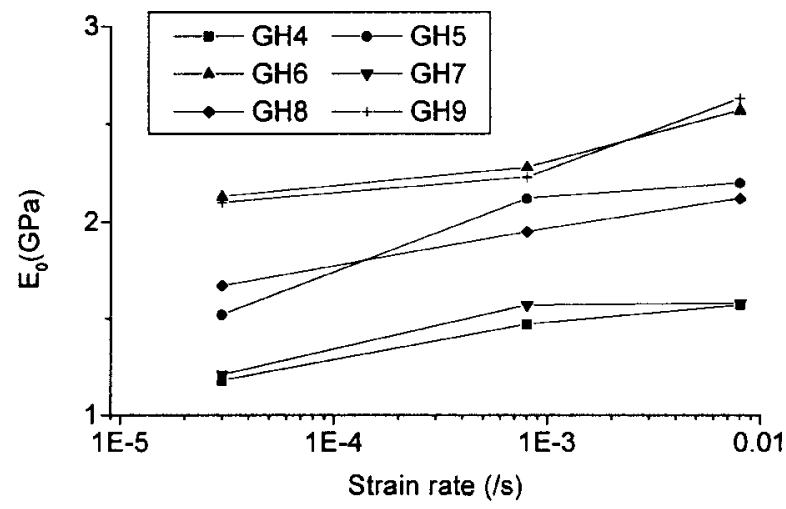

Figure 3. Dependence of the initial modulus $E_{0}$ on strain rate.

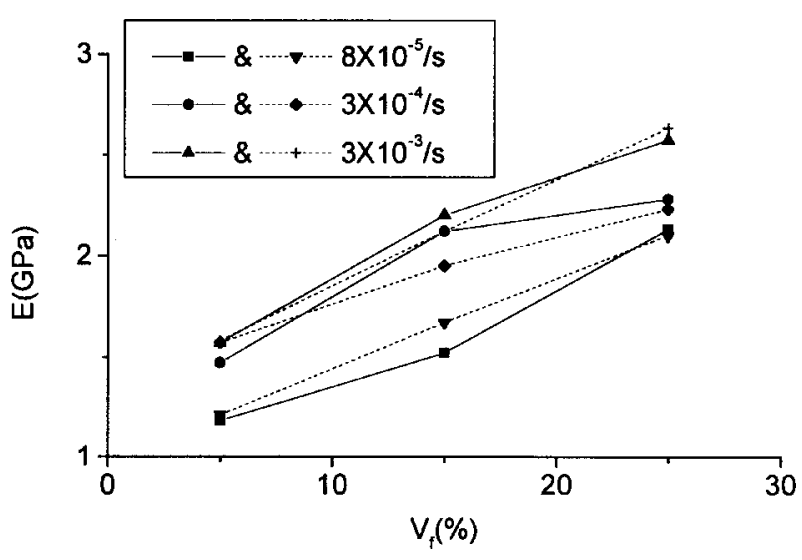

Figure 4. Dependence of the initial modulus $E_{0}$ on GB volume fraction $V_{f}$ - . - . - , strong interfacial adhesion; __, weak interfacial adhesion.

adhesion are always above those for GH4-6 with weak interfacial adhesion, meaning that strong interfacial adhesion can endow the composite with greater resistance to loading. This point of view is the same in the case of fibre-reinforced composites, while the influence of interfacial adhesion on the toughening effect is still a problem worth studying.

\subsection{Effect of particle volume fraction and strain rate on the modulus}

Figure 3 gives the dependence of the Young modulus $E_{0}$ on the strain rate $\log \dot{\varepsilon}$. The Young modulus $E_{0}$ was measured with the loading $\sigma-\varepsilon$ curve of the first cycle, so it represents the initial modulus of the studied materials without damage. It was found that $E_{0}$ increases almost linearly with $\log \dot{\varepsilon}$ despite the different GB contents. This dependence of modulus on strain rates is just one of the characteristics of viscoelastic materials.

The modulus always increases with increasing GB volume fraction, because the rigid GB can resist the transverse contraction of the matrix material, and so increase its rigidity. The quasilinear dependence of the Young modulus on GB volume fraction is observed as shown in Fig 4. In fact, by extrapolation to the case of

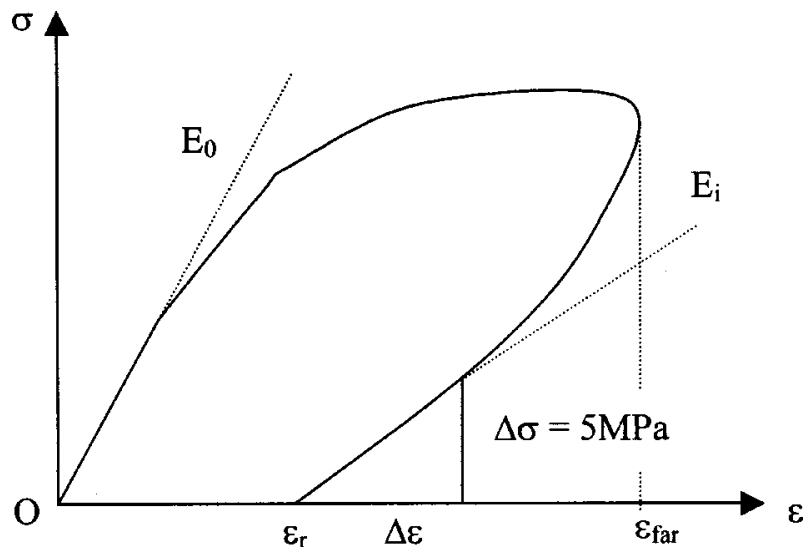

Figure 5. Schematic representation of the damage modulus $E_{\mathrm{i}}$, far-field strain $\varepsilon_{\mathrm{far}}$ and residual strain $\varepsilon_{\mathrm{r}}$. 
pure matrix, ie $V_{\mathrm{f}}=0$, the $E_{0}$ value also respects the quasilinear dependence as shown by the experimental results given by Bai et $a l^{5}$ By using the theory of micromechanics, many studies have been carried out on the prediction of the rigidity of particle-filled materials. ${ }^{13,14}$ However, if the matrix material is neither viscoelastic, nor linear elastic, theoretical modelling becomes very complicated. Besides, the effect of strain rates should be taken into account in the modelling. The influence of the interfacial bonding strength on the modulus is small because modulus measurement is, in general, undertaken under small deformations, and the interface is not debonded

\subsection{Damage evolution and affecting factors}

From cyclic $\sigma-\varepsilon$ curves, it can be seen that the unloading curves incline more and more with the cycles. The damage in the materials nucleates when the applied stress or strain reaches a critical value, and then it accumulates during the cyclic loading-unloading process. The unloading curves at low stress level are linear, not like the initial parts of the loading and unloading curves which are non-linear. Thus, the slope of the unloading curves at low stress can be measured and assumed to represent the modulus of the materials damaged. The stress domain for the modulus measurement is $0-5 \mathrm{MPa}$. Therefore, the Young modulus after certain loading-unloading cycles is $E_{\mathrm{i}}=\Delta \sigma / \Delta \varepsilon=5 / \Delta \varepsilon$, with $\Delta \varepsilon$ depending on the material properties and strain rates. The schematic illustration of the damage modulus $E_{\mathrm{i}}$, far-field strain $\varepsilon_{\text {far }}$ and residual strain $\varepsilon_{\mathrm{r}}$ is shown in Fig 5 . Here, $\varepsilon_{\text {far }}$ is the maximum applied strain of each cycle and $\varepsilon_{\mathrm{r}}$ is the residual strain after unloading to zero.

To quantify the degree of damage which has occurred in the materials, the traditional concept of
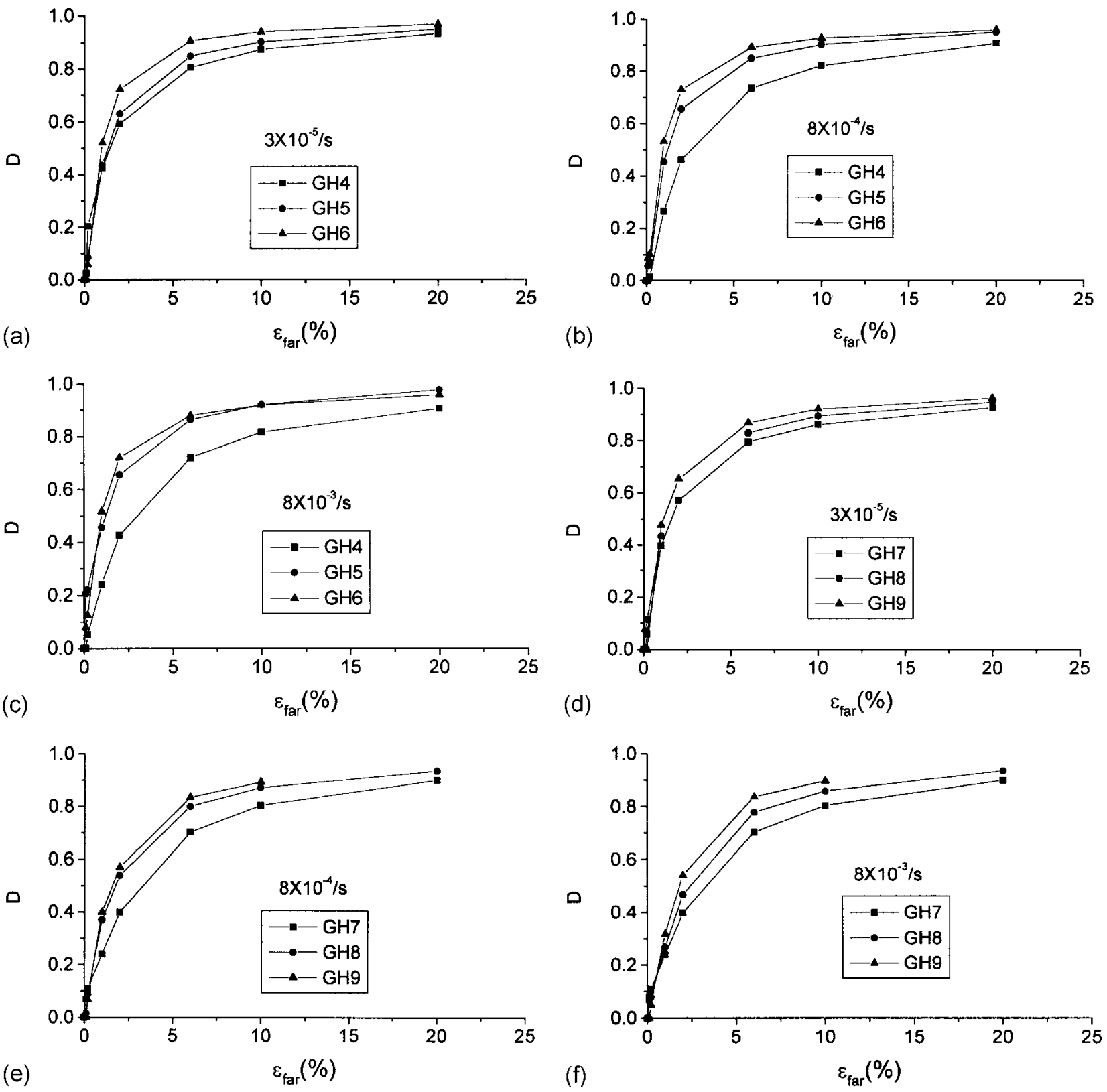

Figure 6. Damage parameter $D$ as a function of far-field strain $\varepsilon_{\mathrm{far}}$. 
damage mechanics is used here by defining the damage parameter $D=1-E_{\mathrm{i}} / E_{0}$. The dependence of the damage parameter $D$ on the far-field strain $\varepsilon_{\mathrm{far}}$ is given in Fig 6 for all the materials studied. The $\mathrm{D}-\varepsilon_{\text {far }}$ curves are composed of two different parts, an initial part of rapid damage development and second part of slow damage development. It seems that the $D$ value will approach unity if the far-field strain increases further.

The influence of GB volume fraction on the damage can be seen from the curves in Fig 6 . For a given $\varepsilon_{\mathrm{far}}$ and under a certain strain rate, the greater the GB volume fraction, the greater the degree of damage. The $\mathrm{GB}$ is considered to be rigid relative to the HDPE matrix, so neither deformation nor rupture happens to GBs. Therefore, the damage may come from matrix microcrazing or interface debonding. It is well known that $\mathrm{HDPE}$ has a glass transition temperature ranging from $-20^{\circ} \mathrm{C}$ to $-100^{\circ} \mathrm{C}$, so at room temperature under which all tests were undertaken, no microcrazing phenomenon takes place during deformation. Besides, the greater the crystallinity, the stiffer the material, ie the movement of the molecular chains is more constrained than in amorphous plastics. It was found that the degree of crystallinity increases with increasing content of fillers. ${ }^{8}$ According to the observation during in situ tensile tests under scanning electron microscopy, interfacial debonding is the source of the damage nucleation as shown by the photograph in Fig 7. This means that with the increasing GB volume fraction, the number of interfacial cracks increases. The damage parameter $D$ is, in fact, a function of the crack density inside the materials. $D$ increases with the crack density, while the latter is directly proportional to the particle volume fraction if interfacial debonding dominates the damage process.

From the curves in Fig 8, the roles played by

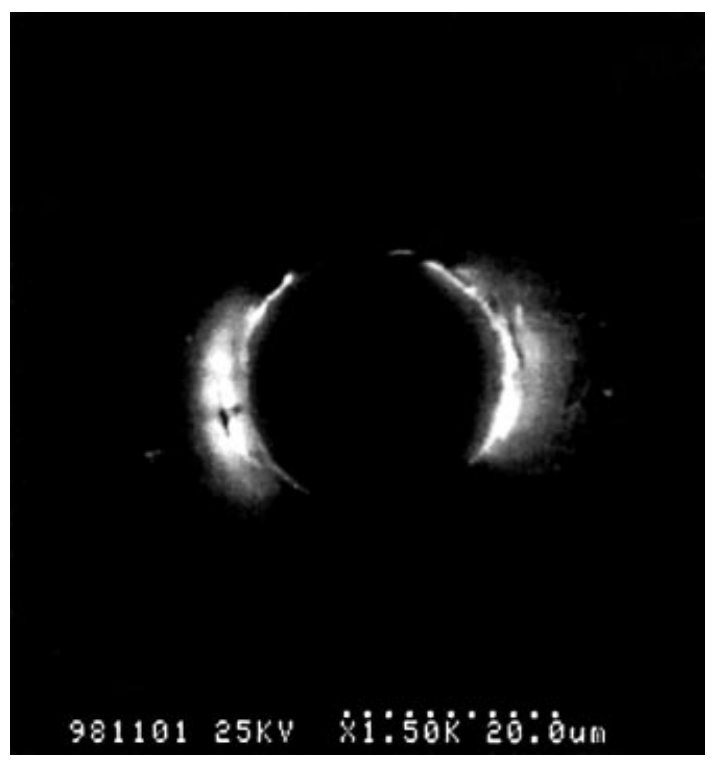

Figure 7. Photograph showing interfacial debonding. interfacial adhesion and strain rates on the damage can be clarified. Under a given strain or stress, the stronger the interfacial adhesion, the lower the number of particles debonded, so the smaller the value of $D$. When $\varepsilon_{\text {far }}$ increases to a critical value, all the particles are debonded from the matrix. From this stage onwards, two materials with the same GB volume fraction but different interfacial adhesion strengths are now both filled with the same density of microcracks. Therefore, $D-\varepsilon_{\text {far }}$ curves for different materials become closer and closer as $\varepsilon_{\text {far }}$ continues to increase.

The $D-\varepsilon_{\text {far }}$ relation in Figs 6 and 8 is different from that for ductile metals. For the latter, the $D$ value generally increases slowly at low stress or strain levels and then increases rapidly at high stress or strain levels until failure. This type of variation of $D$ is due to the damage mechanism being dominated by microcracks.
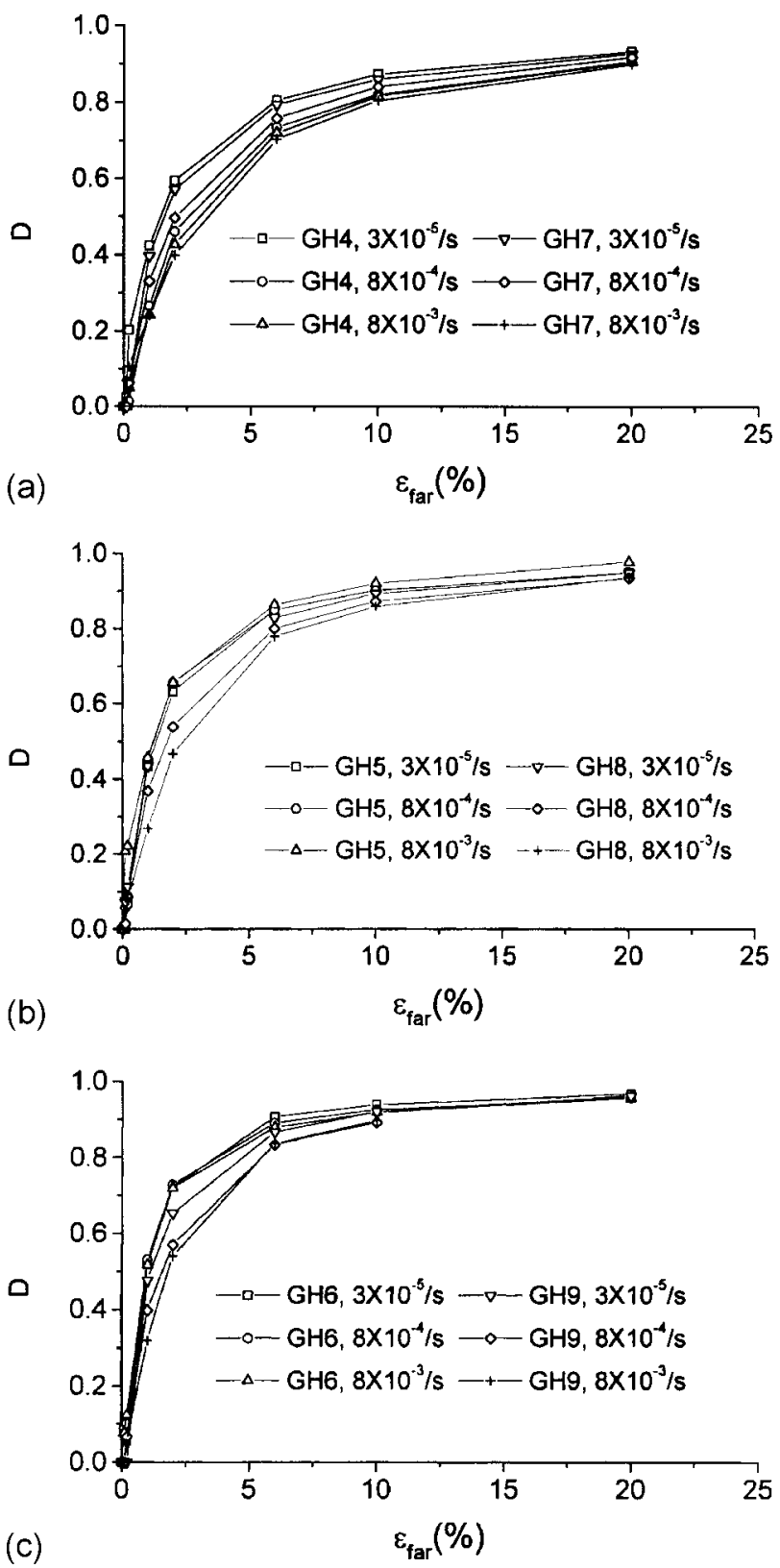

Figure 8. Strain rate and interfacial effect on the damage. 
For a given particle-polymer system, the interfacial bonding strength can be regarded as constant. Hence, when the applied stress is high enough to cause interfacial debonding (ie local normal stress at the interface being equal to the interfacial tensile strength), all particles may theoretically be debonded at the same time. This process of interfacial debonding is brutal and rapid, and is the reason for the rapid increase of the $D$ value in the initial stages of damage. In reality, many particles, but not all, were debonded in the early stages of damage. The slowly increasing part of the $D$ value at high far-field strain is controlled by the growth of interfacial cracks into microvoids formed at two poles of each particle. It was proved in our previous works that before the maximum applied far-field stress was reached, all the particles in the materials were debonded. Subsequent application of the load can only cause the growth of the microvoids and no new cracks are created. It is well known that the damage is sensitive to the density of microdefects, and insensitive to their dimensions. The analysis made here can well explain the $D-\varepsilon_{\text {far }}$ relation.

For a given $\mathrm{GB}$ volume fraction and interfacial adhesion strength, the effect of strain rate on the damage can also be analysed based on the curves shown in Fig 8. Although this effect is not as important as the GB content and interfacial adhesion strength over the range of strain rate used, it seems that a low strain rate can result in greater development of the damage. This may be due to the time-dependent evolution of the damage.

After each loading-unloading cycle, the applied strain does not return to zero, but to a residual strain $\varepsilon_{\mathrm{r}}$ as shown in Fig 5 . The residual strain $\varepsilon_{\mathrm{r}}$ can be
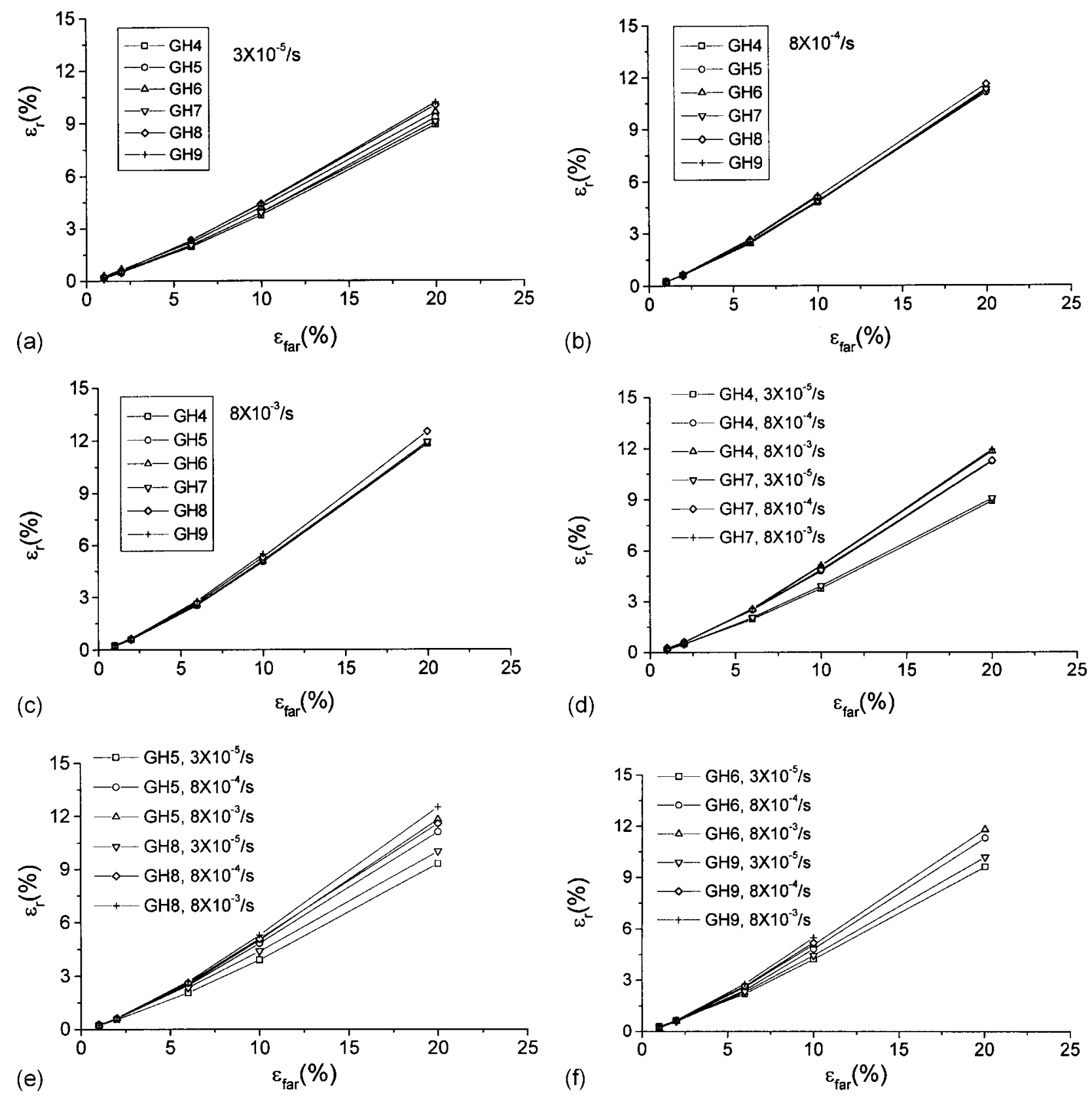

Figure 9. Residual strain $\varepsilon_{\mathrm{r}}$ as a function of far-field strain $\varepsilon_{\text {far }}$. 
measured as a function of far-field strain $\varepsilon_{\text {far }}$, as shown in Fig 9. There is no evident change of the residual strain for different GB volume fractions upon comparing the $\varepsilon_{\mathrm{r}}-\varepsilon_{\text {far }}$ curves of the materials with the same GB volume fraction, but different interfacial adhesion strengths, it seems that the residual strain is independent of the interfacial adhesion strength. However, the effect of strain rate on the residual strain is evident. The greater the strain rate, the greater the residual strain. The residual strain is composed of two parts: residual deformation of the microvoids still open and viscoelastic strain. The viscoelastic strain depends on the reverse time. As the strain rate increases, the reverse time of the matrix becomes shorter, resulting in greater residual strain. This point of view corresponds well with the experimental results. The interfacial microvoids continue to grow with loading at two poles of the particles. During unloading, the microvoids are compressed to reduce their dimensions, but not completely closed as assumed.

\section{CONCLUSIONS}

Rigid particle filled polymer composites are designed to exhibit both high strength and high toughness, but these properties are strongly affected by the particle size and size distribution, particle content and interfacial adhesion strength. Their mechanical properties also depend on the strain rates because they are, in general, viscoelastic. The damage during cyclic tension was found to be controlled by interfacial debonding. Strong interfacial adhesion can successfully postpone damage nucleation. A high particle volume fraction can result in an increase of the rigidity. Due to the damage mechanisms induced by interfacial debonding, the greater the particle volume fraction, the greater the density of microcracks formed at the interface. The effect of the strain rates on the damage is time-dependent, ie a low strain rate favours damage development. The residual strain is related to the residual deformation of the microvoids formed at two poles of the particles.

\section{ACKNOWLEDGEMENT}

This work was supported by the National Natural Science Foundation of China (grants 19872007 and 10032010).

\section{REFERENCES}

1 Kurauchi T and Ohta T, f Mater Sci 19:1699-1709 (1984).

2 Schreiber HP and St Germain F, $\mathcal{F}$ Adhes Sci Technol 4:319 (1990).

3 Bai SL, Chen JK, Huang ZP and Lu ZD, Key Eng Mater 183187:1075-1080 (2000).

4 Bai SL, Chen JK, Huang ZP and Liu ZD, Polym Int 50:222-228 (2001).

5 Bai SL, Cao K, Chen JK and Liu ZD, Polym Polym Composites 8:1-6 (2000).

6 Lemaitre J, A Course on Damage Mechanics, Springer, Berlin (1992).

7 Vollenberg PHT and Heikkens D, Polymer 30:1656 (1986).

8 Barttczak Z, Argon AS, Cohen RE and Weinberg M, Polymer 40:2347-2365 (1999).

9 Bai SL, Chen JK, Huang ZP and Yu ZZ, J Mater Sci Lett 19:1587-1589 (2000).

10 Rothon R, Particulate-Filled Polymer Composites, Longman, Harlow (1995).

11 Tzika PA, Boyce MC and Parks DM, f Mech Phys Solids 48:1893-1929 (2000).

12 Socrate S and Boyce MC, $\mathcal{F}$ Mech Phys Solids 48:233-273 (2000).

$13 \mathrm{Hu}$ GK and Weng GJ, Mech Mater 32:495-503 (2000).

14 Bergstrom JS and Boyce MC, Rubber Chem Technol 72:633-656 (2000). 